\title{
Journalof the American Chemical Society
}

\section{Communication}

Nanometer Arrays of Functional Light Harvesting Antenna Complexes by Nanoimprint Lithography and Host\#Guest Interactions

Maryana Escalante, Yiping Zhao, Manon J. W. Ludden, Rolf Vermeij, John D. Olsen, Erwin Berenschot, C. Neil Hunter, Jurriaan Huskens, Vinod Subramaniam, and Cees Otto J. Am. Chem. Soc., 2008, 130 (28), 8892-8893 • DOI: 10.1021/ja802843m • Publication Date (Web): 21 June 2008

Downloaded from http://pubs.acs.org on January 6, 2009

\section{More About This Article}

Additional resources and features associated with this article are available within the HTML version:

- Supporting Information

- $\quad$ Access to high resolution figures

- $\quad$ Links to articles and content related to this article

- Copyright permission to reproduce figures and/or text from this article

\section{View the Full Text HTML}




\title{
Nanometer Arrays of Functional Light Harvesting Antenna Complexes by Nanoimprint Lithography and Host-Guest Interactions
}

\author{
Maryana Escalante, ${ }^{\dagger}$ Yiping Zhao, ${ }^{\ddagger}$, Manon J. W. Ludden, ${ }^{\ddagger}$ Rolf Vermeij, ${ }^{\dagger}$ John D. Olsen," \\ Erwin Berenschot, ${ }^{\S}$ C. Neil Hunter," Jurriaan Huskens, ${ }^{*, \neq}$ Vinod Subramaniam, ${ }^{*, \dagger}$ and Cees Otto ${ }^{*, \dagger}$ \\ Biophysical Engineering Group, Molecular Nanofabrication Group, Transducer Science and Technology Group, \\ and MESA+ Institute for Nanotechnology, University of Twente P.O. Box 217, 7500 AE Enschede, The \\ Netherlands, and Department of Molecular Biology and Biotechnology, University of Sheffield, \\ Sheffield S10 2TN, U.K.
}

Received April 17, 2008; E-mail: c.otto@utwente.nl; v.subramaniam@utwente.nl; j.huskens@utwente.nl

Supramolecular interactions play a key role in the functional architecture of nature. On patterned surfaces, interactions can be adjusted in strength and further modulated by the orientation of target molecules ${ }^{1,2}$ Here, we have engineered functional ordered arrays of purified components of the photosynthetic system. We relied on multivalent interactions to drive the selective assembly of functional light harvesting LH2 antenna complex onto nanometer structured $\beta$-cyclodextrin $\left(\beta\right.$-CD) monolayers ${ }^{2}$ patterned by nanoimprint lithography (NIL). ${ }^{3,4}$

The nanomachinery of the photosynthetic bacterium Rhodobacter sphaeroides has been an invaluable model for the study of biophysics, biochemistry, and molecular biology of photosynthesis. ${ }^{5}$ The membrane-bound LH2 complex is built of nine identical subunits each consisting of an $\alpha$ and a $\beta$ polypeptide. A total of 27 bacteriochlorophyll (BChl) molecules (18 BChl B850 and 9 B800) are bound to this structure having dimensions of $\sim 6 \mathrm{~nm}$ in height and $\sim 6 \mathrm{~nm}$ in diameter. ${ }^{6,7} \mathrm{LH} 2$ are interesting candidates for applications in synthetic light converting circuits because of their well-defined optical properties, such as a broad spectral range, high absorption cross section, efficient energy transfer ${ }^{8}$ and high photostability. Photosynthetic antenna systems have been used in studies exploiting covalent ${ }^{9}$ and electrostatic interactions ${ }^{10}$ to promote attachment to a chemically defined surface. A major challenge remains in the control of the interfacial properties and the associated multiple weak interactions to produce and optimize organized molecular structures with controlled directional energy migration.

Here, we show an approach based on a combination of sitedirected mutagenesis, NIL and multivalent host-guest interactions. LH2 complexes were engineered with cysteine residues at the penultimate position of the C-terminus of the $\alpha$ polypeptide chain. These strategic positions at the periplasmic face ensured the orientation of all of the protein complexes upon binding to the surface. The cysteine residues were modified with iodoacetyltri(ethylene glycol) mono(adamantyl ether), AdI, block 3, Chart 1. Protein aggregates in an aqueous buffered solution of $20 \mathrm{mM}$ HEPES, $\mathrm{pH} 8.0,0.03 \% n$-dodecyl- $\beta$-D-maltoside ( $\beta$-DDM) were mixed in 1:20 mol equiv with the AdI in $1.3 \%$ dimethyl sulfoxide (DSMO) to yield an average of three adamantyl molecules linked to each protein complex, hereafter referred to as $\mathrm{Ad}_{n} \mathrm{LH} 2{ }^{1{ }^{11}}$

When adsorbing $\mathrm{Ad}_{n} \mathrm{LH} 2$ onto a $\beta \mathrm{CD}$-coated glass substrate (Chart 1, block 1), hexa(ethylene glycol)mono(adamantyl ether) AdHEG, block 2, served as a temporary blocking agent for the

\footnotetext{
† Biophysical Engineering Group, University of Twente.

* Molecular Nanofabrication Group, University of Twente.

$\$$ Transducer Science and Technology Group, University of Twente.

"University of Sheffield.
}

Chart 1. Representation of Host, Guest, and Target Molecules ${ }^{a}$
1

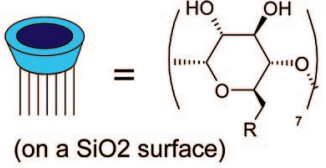

3

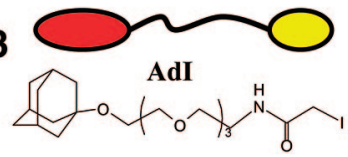

2
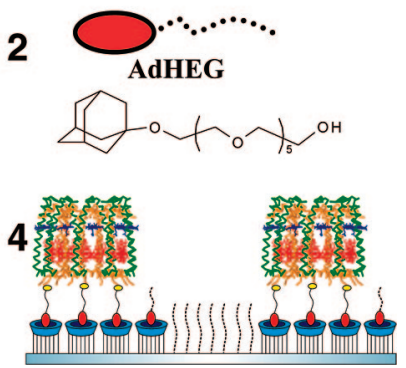

${ }^{a}$ (1) $\beta$-CD heptamine, host molecule, ${ }^{2}$ (2) Hexa(ethylene glycol)mono(adamantyl ether) (AdHEG), (3) iodoacetyl-tri(ethylene glycol)) mono(adamantyl ether), (AdI), (4) $\mathrm{Ad}_{n} \mathrm{LH} 2$ on the $\beta$-CD monolayer.

$\beta$-CD cavities, preventing nonspecific adsorption by shielding the surface with the HEG chain. ${ }^{12}$ The monovalent AdHEG is later effectively displaced through competition by exploiting the higher affinity of the multivalent $\mathrm{Ad}_{n} \mathrm{LH} 2 .{ }^{12}$ The assembly on the patterned surface is depicted in block 4.

We used a custom-built hybrid high resolution scanning probespectral microscope ${ }^{13}$ to characterize the patterned proteins in liquid conditions. A fluorescence titration allowed us to simultaneously address the optical properties after modification of the LH2 complex and the specificity of the binding on nonpatterned $\beta$-CD surfaces. Upon excitation of the $\mathrm{LH} 2$ complexes via the $\mathrm{B} 800 \mathrm{BChl}$ (donor), the energy is then transferred within the complex to the B850 (acceptor) and ultimately emitted as fluorescence. A dilute solution of nonmodified LH2 complexes was incubated onto the $\beta$-CD monolayer and rinsed with buffer. The average emission spectra (blue box trace, Figure 1a), indicates a high contribution of nonspecific adsorption. Subsequently, after pretreating the surface with $1 \mathrm{mM}$ solution of AdHEG, a solution of nonmodified LH2 complexes in $1 \mathrm{mM}$ AdHEG was incubated onto the substrate. The nonspecific adsorption was reduced by $94 \%$ (open box trace). The previous experiment was repeated with $\mathrm{Ad}_{n} \mathrm{LH} 2$ complexes. The increase in intensity of the emission signal (green star trace) reveals that the protein complexes specifically bind to the surface by replacement of the monovalent AdHEG with the multivalent $\mathrm{Ad}_{n} \mathrm{LH} 2$. This replacement ${ }^{12}$ and the observed stability against rinsing with a $\beta C D$ solution ${ }^{2}$ are evidence for the formation of stable multivalent complexes via at least 3 Ad linkers. Quantitatively, the spectral response from the immobilized $\mathrm{Ad}_{n} \mathrm{LH} 2$ complexes showed no visible shift of the emission maximum, $\sim 868 \mathrm{~nm}$, with respect to bulk measurements of nonmodified LH2 complexes (red triangle trace). This observation is compelling evidence that the complete procedure of labeling and surface adsorption has maintained the 

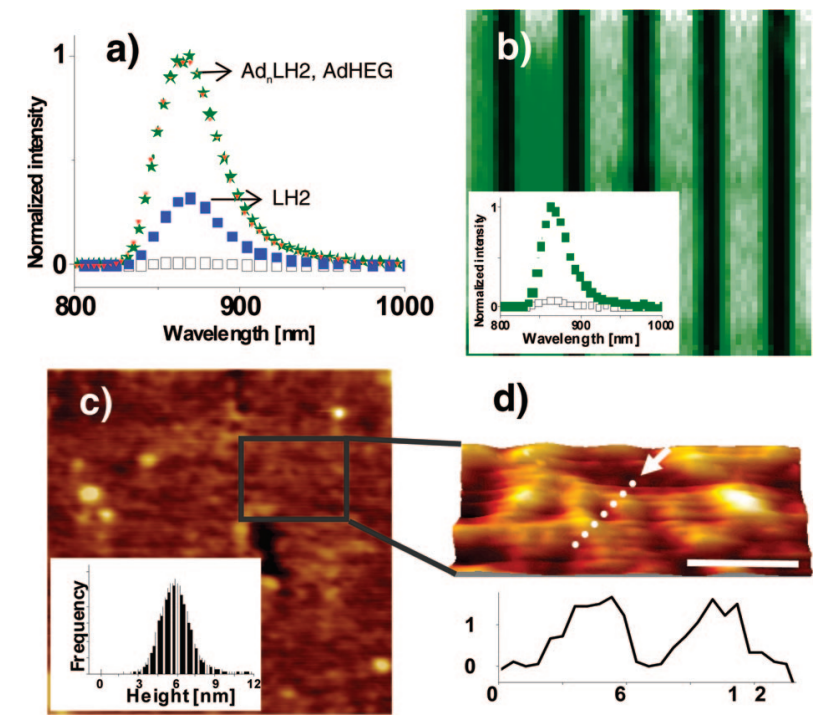

d)

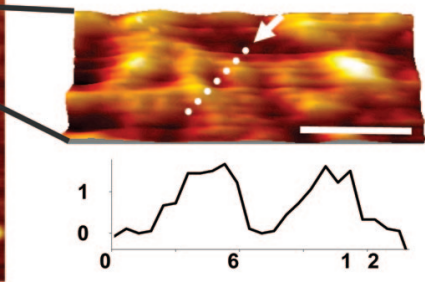

Figure 1. (a) Fluorescence titration: nonlabeled LH2 (blue box); nonlabeled LH2, 1 mM AdHEG (open box); $\mathrm{Ad}_{n} \mathrm{LH} 2,1 \mathrm{mM}$ AdHEG (green star); reference spectrum of nonlabeled LH2 in solution (red triangle). (b) False color, fluorescent-spectral image of $\mathrm{Ad}_{n} \mathrm{LH} 2$ patterns $(\beta$-CD/PEG), $40 \times$ $40 \mu \mathrm{m}, 64 \times 64$ pixels; inset shows emission spectra active area (green box), passivated (open box). (c) AFM topography in liquid, $150 \times 150 \mathrm{~nm}$ area, $256 \times 256$ pixels, inset shows histogram height distribution. (d) Section across a LH2 complex showing a profile along the dotted line; scale bar, $10 \mathrm{~nm}$.

structural integrity of the LH2 membrane protein. Figure $1 \mathrm{~b}$ shows a representative spectral image for the patterned $\mathrm{Ad}_{n} \mathrm{LH} 2$ complexes. On each pixel, a full spectrum was recorded, and integrated over the respective emission band of the $\mathrm{LH} 2$ complexes. The exposure of patterns of $\beta$-CD SAMs surrounded by a protein resistant PEG $\mathrm{SAM}^{14}$ to the solution of $\mathrm{Ad}_{n} \mathrm{LH} 2,1 \mathrm{mM}$ AdHEG resulted in the selective assembly of the protein onto the $\beta$-CD regions (green) in a ratio of 16:1 as indicated by the averaged emission spectra (inset Figure 1b) from active and passivated areas. A monolayer coverage was suggested by quantitative spectral images, which revealed only minor variations $(<5 \%)$ in intensity over the patterned area.

We performed AFM imaging at low tapping amplitudes to assess the density of the putative monolayer. Figure 1c shows an AFM height image of the patterned LH2 complexes; analysis of the surface indicates a uniform height of $\sim 6 \mathrm{~nm}$ (inset) with reference to the defect (black region), suggesting monolayer coverage of the protein. In the AFM image (Figure 1d) ring-shaped LH2 proteins could be observed, which are attributed to the exposed face of the complexes. The height histogram (inset Figure 1c) indicated less than $1 \%$ multilayer stacked aggregates of proteins in agreement with the fluorescence images.

In an attempt to prepare structures approaching molecular dimensions, NIL was performed using stamps with silicon ridges as small as $40 \mathrm{~nm}, 4 \mu \mathrm{m}$ period. Figure $2 \mathrm{a}$ shows an AFM height image in liquid of $\operatorname{Ad}_{n} \mathrm{LH} 2$ complexes on a $\beta$-CD SAM. The mean fwhm of the lines after processing is $80 \pm 5 \mathrm{~nm}$ with a height of $\sim 6 \pm 1 \mathrm{~nm}$ consistent with the assembly of a monolayer of LH2 complexes, Figure 2c. The increase in width, relative to that of the NIL stamp, is attributed to the process of removal of the residual layer in the imprinting process. Figure $2 b$ shows a fluorescence image acquired with a single photon counting avalanche photodiode, with intensity variations of $\pm 14 \%$ along the lines. The apparent
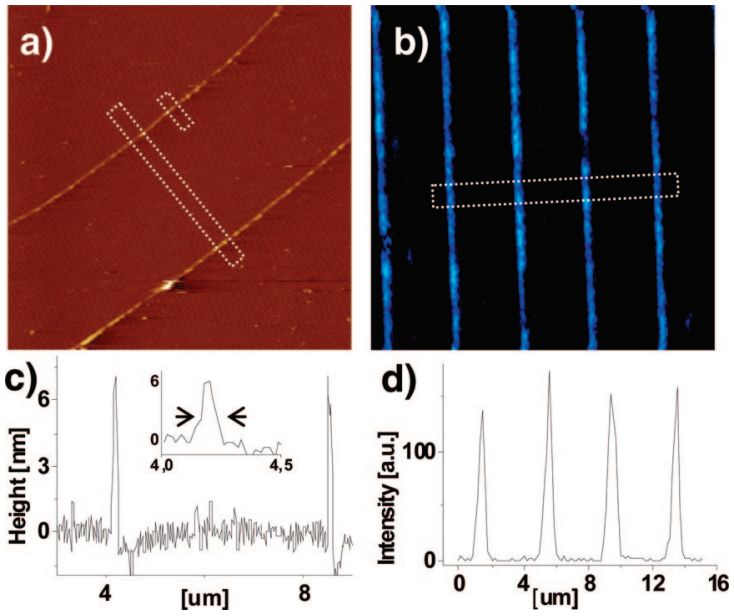

Figure 2. (a) AFM topography in liquid of $\mathrm{Ad}_{n} \mathrm{LH} 2 \beta$-CD/PEG SAM, 10 $\times 10 \mu \mathrm{m}$, z-scale $30 \mathrm{~nm}$, and respective cross section (c), fwhm of $80 \mathrm{~nm}$ (inset). (b) False color fluorescent image. (d) Cross section of panel b. width of these structures (Figure 2d) is defined by the optical resolution of $\sim 700 \mathrm{~nm}$.

We have achieved exquisite spatial control at different length scales of functional specifically bound LH2 complexes in a high throughput manner by exploiting host-guest interactions and NIL. In situ characterization of the formation of these assemblies at molecular dimensions and the fabrication of mixed protein arrays are the subject of current research.

Acknowledgment. This work was supported by NanoNed TMM.7124; NWO-CW (M.J.W.L.; Vidi Vernieuwingsimpuls 700.52.423 to J.H.); MESA+ (SRO-NF) (Y.Z.); C.N.H. and J.D.O. acknowledge funding from the BBSRC, U.K.

Supporting Information Available: Materials and experimental procedures. This material is available free of charge via the Internet at http://pubs.acs.org.

\section{References}

(1) Mulder, A.; Onclin, S.; Peter, M.; Hoogenboom, J. P.; Beijleveld, H.; ter Maat, J.; Garcia-Parajo, M. F.; Ravoo, B. J.; Huskens, J.; van Hulst, N. F.; Reinhoudt, D. N. Small 2005, 1, 242-253.

(2) Ludden, M. J. W.; Reinhoudt, D. N.; Huskens, J. J. Chem. Soc. Rev. 2006, 35, 1122-1134.

(3) Chou, S. Y.; Krauss, P. R.; Renstrom, P. J. Science 1996, 272, 85-87

(4) Maury, P.; Peter, M.; Crespo-Biel, O.; Ling, X. Y.; Reinhoudt, D. N.; Huskens, J. Nanotechnology 2007, 18, 044007.

(5) Hunter, C. N.; Tucker, J. D.; Niederman, R. A. Photochem. Photobiol. 2005, 4, 1023-1027.

(6) Scheuring, S.; Seguin, J.; Marco, S.; Levy, D.; Breyton, C.; Robert, B.; Rigaud, J. L. J. Mol. Biol. 2003, 325, 569-580.

(7) Bahatyrova, S.; Frese, R N.; van der Werf, K. O.; Otto, C.; Hunter, C. N.; Olsen, J. D. J. Biol. Chem. 2004, 279, 21327-21333.

(8) (a) Sundstrom, V.; Pullerits, T.; van Grondelle, R. J. Phys. Chem B 1999, 103, 2327-2346. (b) van Grondelle, R.; Novoderezhkin, V. I. Phys. Chem. Chem. Phys. 2006, 8, 793-807.

(9) Reynolds, N. P.; Janusz, S.; Escalante-Marun, M.; Timney, J.; Ducker, R. E.; Olsen, J. D.; Otto, C.; Subramaniam, V.; Leggett, G. J.; Hunter, C. N. J. Am. Chem. Soc. 2007, 129, 14625-14631.

(10) Escalante, M.; Maury, P.; Bruinink, C. M.; van der Werf, K.; Olsen, J. D.; Timney, J. A.; Huskens; J.; Hunter, C. N.; Subramaniam, V.; Otto, C. Nanotechnology 2008, 19, 025101.

(11) Molecular Probes, Invitrogen Corporation, Carlsbad, CA.

(12) Ludden, M. J. W.; Mulder, A.; Tampe, R.; Reinhoudt, D. N.; Huskens, J. Angew.Chem., Int. Ed. 2007, 46, 4104-4107.

(13) Kassies, R.; Van der Werf, K. O.; Lenferink, A.; Hunter, C. N.; Olsen, J. D.; Subramaniam, V.; Otto, C. J. Microsc. (Oxford) 2005, 217, 109116.

(14) Kannan, B.; Castelino, K.; Chen, F.; Majumdar, A. Biosens. Bioelectron. 2006, 21, 1960-1967.

JA802843M 